\title{
Storia naturale dei noduli tiroidei benigni
}

\author{
Michele Marinò ${ }^{1}$
}

Pubblicato online: 2 luglio 2015

C Springer International Publishing AG 2015

\section{Commento a:}

The natural history of benign thyroid nodules.

C. Durante, G. Costante, G. Lucisano, R. Bruno,

D. Meringolo, A. Paciaroni, E. Puxeddu, M. Torlontano,

S. Tumino, M. Attard, L. Lamartina, A. Nicolucci,

S. Filetti.

JAMA (2015) 313(9):926-935

In questo importante studio veniva valutata la frequenza e l'entità delle variazioni dimensionali dei noduli tiroidei. Venivano inoltre identificati alcuni fattori che si associano a tali variazioni nonché la frequenza di sviluppo di nuovi noduli e di cancro. Le basi dello studio erano la mancanza di un consenso adeguato sul follow-up ottimale dei noduli tiroidei in un'epoca in cui, grazie all'impiego diffusissimo dell'ecografia, la frequenza con cui essi vengono diagnosticati è elevatissima, specie nelle aree geografiche iodo-carenti.

Si trattava di uno studio prospettico, multicentrico, osservazionale, condotto in 992 pazienti consecutivi con noduli tiroidei asintomatici, in numero da 1 a 4 , con caratteristiche ecografiche o citologiche di benignità. I pazienti venivano reclutati in otto diversi centri di riferimento italiani e venivano osservati per un periodo di 5 anni. I pazienti venivano sottoposti a controlli ecografici annuali e veniva considerato significativo un aumento di dimensioni di almeno il $20 \%$ in almeno due diametri dei noduli, con un aumento minimo di
$2 \mathrm{~mm}$ (end-point primario). Venivano valutati vari parametri basali possibilmente associati alla crescita nodulare. Gli end-point secondari erano lo sviluppo di nuovi noduli e la diagnosi di cancro della tiroide durante il follow-up.

Veniva osservata una crescita dei noduli in 153 pazienti (15,4\% [95\% CI, 14,3-16,5\%]). 174 dei 1567 noduli originali $(11,1 \%$ [95\% CI, 10,3-11,9\%]) aumentavano di dimensioni, con un incremento medio del diametro maggiore di 4,9 $\mathrm{mm}$. La crescita dei noduli era associata alla presenza di noduli multipli, al sesso maschile, mentre rappresentava un fattore protettivo un'età maggiore o uguale a 60 anni ed era, invece, un fattore di rischio un'età minore di 45 anni. Il cancro della tiroide veniva diagnosticato in 5 noduli originali $(0,3 \%$ [95\% CI, $0,0-0,6 \%])$, di cui solo 2 erano cresciuti durante il follow-up. Veniva osservato lo sviluppo di nuovi noduli in 93 pazienti $(9,3 \%$ [95\% CI, 7,5-11,1\%]), con rilievo di cancro in uno di essi.

Questo studio dimostra che in presenza di noduli tiroidei asintomatici, con caratteristiche di benignità all'ecografia o alla citologia, la maggior parte di essi non mostra una crescita significativa in un periodo di 5 anni. Inoltre, lo sviluppo di cancro è estremamente raro, mentre non è infrequente lo sviluppo di nuovi noduli benché, tuttavia, nella quasi maggioranza dei casi benigni. I risultati di questo studio hanno grande importanza e rappresentano una solida base per la gestione dei noduli tiroidei nella pratica clinica.

M. Marinò

michele.marino@md.unipi.it

1 U.O. Endocrinologia 1, Università di Pisa, Pisa, Italia 